\title{
The Role of Antibiotic Prophylaxis in the Ureteroscopy Treatment for Ureteral Lithiasis
}

\author{
Maier $\mathrm{A}^{1,2}$, Vida $\mathrm{O}^{1,2}$, Szollosi $\mathrm{A}^{1,2}$, Golea $\mathrm{O}^{1,2}$, Todea $\mathrm{C}^{1,2}$, Boja $\mathrm{R}^{1,2}$, Martha Orsolya ${ }^{1,2}$ \\ 1 Department of Urology, County Emergency Clinical Hospital, Tîrgu Mureș, Romania \\ 2 University of Medicine and Pharmacy, Tîrgu Mureș, Romania
}

\begin{abstract}
Introduction: One of the most common pathologies in urological praxis is urinary lithiasis. Extracorporeal shock wave lithotripsy (ESWL) or frequently retrograde ureteroscopy are modern pathways in the treatment of this kind of pathology. There are certain problems which may develop after the ureteroscopy such as infection with fever complication.

Material and method: This retrospective study evaluates 164 patients who underwent ureteroscopy treatment over a period of two years (2011-2012). We compared the infection complication episode (with fever) in 33 (20.12\%) patients with antibiotic prophylaxis (group A) versus 131 (79.87\%) patients without prophylaxis (group B). Antibiotics used for prophylaxis were: amoxicilinum and clavulanic acid, generation I and II cephalosporines.

Results: Twenty-four (14.63\%) patients presented postsurgical fever. Most febrile patients were those with grade II hydronephrosis - 16 (66.66\%), of which 2 (6.06\%) patients from group A and 14 (10.68\%) from group B. From group A, 3 (12\%) patients with stones below $10 \mathrm{~mm}$ had fever, while 18 (14.87\%) from group B developed this complication. Among the patients with stones' size over $10 \mathrm{~mm}, 1$ (14.28) patient from group A and 2 (25\%) patients from group B had fever.

Conclusions: Patients undergoing the ureteroscopy treatment should be investigated before the procedures for the presence of bacteria in order to avoid complications like infection associated with fever. Antibiotic prophylaxis may reduce the incidence of postoperative infection in the ureteroscopy treatment.
\end{abstract}

Keywords: retrograde ureteroscopy, urinary infection, ureteral lithiasis, antibiotic prophylaxis

Received: 11 June 2013 / Accepted: 16 June 2014

\section{Introduction}

Urinary lithiasis is one of the most common pathologies in urological praxis. In most cases the stones are formed in the renal pelvis and then they descend on the urinary pathways, such as the ureter, where they can be enclaved in the mucosa, without spontaneous passage. Nowadays, the treatment of urolithiasis in general, and of ureteral stones in particular, comprises extracorporeal shock wave lithotripsy (ESWL) or endoscopic procedures, such as retrograde ureteroscopy (rigid, semi-rigid and flexible) [1]. The endoscopic treatment is recommended in case of ESWL failure or if stone size exceeds $10 \mathrm{~mm}$ [2]. The treatment of ureteral stones is followed by few complications, one of them is represented by postoperative infections. Thus, we recommend bacteriological investigations for every patient with ureteral stones to prevent postoperative complications, such as infections associated with fever or sepsis. Taking into consideration these situations, it is advisable to follow the 2014 indications of the European Association of Urology, who recommend the administration of antibiotics before surgical interventions on the urinary tract; the recommended antibiotics are: prime intention trimethoprim-sulfamethoxazol as first line treatment, second or third generation cephalosporines, aminopenicillins/BLI, and fluoroquinolones if necessary [3].

Correspondence to: Adrian Maier

E-mail: maier23adrian@yahoo.com
The aim of this study is to evaluate the importance of the antibiotics prophylaxis used for treatment of the uncomplicated ureteral stones.

\section{Material and method}

We evaluated in retrospective the medical information of patients from the Urological Clinic of Tîrgu Mureș, between January 2011 and December 2012, who underwent retrograde ureteroscopy for ureteral lithiasis. The patients presented at our Urological Department with lumbar pain such as colic or nephralgia, having secondary passage stones in different segments of the ureter. In this study we excluded patients with severe infections such as sepsis, with an indwelling ureteral catheter or percutaneous nephrostomy and none of them underwent surgical procedures in acute infection episode (full fever spurt).

We divided the patients into two groups: the first group (A) received prophylactic preoperative treatment $-33 \mathrm{pa}-$ tients $(20.12 \%)$, while the second group (B) did not receive antibiotic prophylaxis - 131 patients $(79.87 \%)$. The antibiotic prophylaxis was performed 24 hours before the intervention. The antibiotics used were amoxicillinum with clavulanic acid, second generation cephalosporines with intravenous administration, for a period of 2-3 days after the operation. We have also evaluated the postoperative condition of the patients in relation to the state of the affected kidney and the grade of hydronephrosis deter- 
Table I. Fever complications (results of postsurgical procedures)

\begin{tabular}{|c|c|c|c|c|c|}
\hline & & & Grade I hydronephrosis & Grade II hydronephrosis & Grade III hydronephrosis \\
\hline \multirow[t]{4}{*}{ Lumbar } & With antibiotics & Fever & 0 & 1 & 2 \\
\hline & & No fever & 2 & 5 & 6 \\
\hline & Without antibiotics & Fever & 0 & 7 & 2 \\
\hline & & No fever & 5 & 15 & 10 \\
\hline \multirow[t]{4}{*}{ Iliac } & With antibiotics & Fever & 0 & 1 & 0 \\
\hline & & No fever & 0 & 0 & 1 \\
\hline & Without antibiotics & Fever & 0 & 3 & 0 \\
\hline & & No fever & 0 & 8 & 5 \\
\hline \multirow[t]{4}{*}{ Pelvic } & With antibiotics & Fever & 0 & 0 & 2 \\
\hline & & No fever & 2 & 6 & 5 \\
\hline & Without antibiotics & Fever & 1 & 4 & 1 \\
\hline & & No fever & 17 & 35 & 17 \\
\hline
\end{tabular}

mined by the lithiasic obstacle. The patients have been further divided into two groups, depending on the severity of cases: the first group consisted of patients with slight to moderate affections and the second group of patients with severe affections. This division was made in order to evaluate the role of antibiotic prophylaxis in relation to the degree of the renal damage in the postoperative evolution.

\section{Results}

We have detected a number of $56(34.13 \%)$ calculi at lumbar level, $18(10.97 \%)$ calculi at iliac level and 90 $(54.87 \%)$ at pelvic level. Based on their dimensions, they have been divided into two groups: up to $10 \mathrm{~mm}-146$ cases $(89.02 \%)$ and over $10 \mathrm{~mm}$ a number of $18(10.97 \%)$ cases. From the total of 164 patients on whom we performed retrograde ureteroscopy, 24 (14.64\%) have presented a postoperative febrile state.

The incidence of reported febrile cases to the hydronephrosis occured as follows: for grade II hydronephrosis we had $16(18.6 \%)$ febrile patients, of which $2(15.38 \%)$ patients from the antibiotic prophylaxis group; the rest of 14 (19.17\%) patients did not benefit from antibiotic prophylaxis, and we had 7 febrile patients with grade III hydronephrosis (Table I). The stones with dimensions of up to 10 $\mathrm{mm}$ caused the onset of postoperative fever in $21(87.5 \%)$ patients, and the ones with dimensions of over $10 \mathrm{~mm}$ in $3(12.5 \%)$ patients. In febrile patients with stones up to $10 \mathrm{~mm}, 3$ (12\%) patients followed prophylactic treatment, the other $18(14.87 \%)$ patients were without prophylactic treatment. In the case of the second group with calculi' size of over $10 \mathrm{~mm}$, the fever occurred in the group without antibiotic treatment in $2(25 \%)$ patients, while in the group with antibiotic treatment the fever was present in only 1 (14.28\%) patient (Table II). Infectious complications were

Table II. Results depending on the size of stones

\begin{tabular}{lcccc}
\hline & \multicolumn{2}{c}{ With prophilaxy } & \multicolumn{2}{c}{ Without prophilaxy } \\
\hline & Fever & No fever & Fever & No fever \\
\hline Stones up to $10 \mathrm{~mm}$ & 3 & 22 & 18 & 103 \\
Stones over $10 \mathrm{~mm}$ & 1 & 7 & 2 & 8 \\
\hline
\end{tabular}

caused by the most common germ E. coli, present in 17 $(70.83 \%)$ cases, the remaining germs being represented by Enteroccocus spp. 3 (12.5\%), Proteus 1 (4.16\%), Pseudomonas aeruginosa 3 (12.5\%).

\section{Discussion}

Previous studies evaluated the post procedural complications after ureteroscopy in general, while in our study we evaluated the infectious episodes in particular; thus we aimed to demonstrate the importance of knowing the risk of infections in patients without antibiotic prophylaxis treatment who underwent endoscopic procedures. The gender repartition emphasized a slightly higher proportion in males, the ratio being $1.07 / 1$, suggesting similar results to previous studies [5].

According to the 2013 guidelines of the European Association of Urology, ureteroscopy is recommended in the following situations: stones with low chance of spontaneous passage, persistent pain and obstruction despite adequate analgesic medication, and in case of renal insufficiency (renal distress, bilateral obstruction, single kidney) [2]. Ureteroscopy proved its utility over the years in the case of lithiasic affections of the superior urinary tract, having only minor postoperative complications. Endoscopic interventions on the superior urinary tract can have complications such as infections manifested through the presence of febrile syndrome [4,5], making the use of antibiotic treatment notable at least for discussion. Postoperative infectious complications depend both on the surgeon's experience (best time surgery, surgical technique), and the patient's history, urinary tract infection episodes, as well as the preoperative presence of symptomatic/asymptomatic bacteriuria. Fever as a sign of postoperative infection episode is met in few cases, Geavlete reporting an incidence of $1.13 \%$ of total cases [5]. Thus, we recommend bacteriological investigations for every patient with ureteral stones to prevent or to understate the postoperative complications such as infections associated with fever or sepsis.

Therefore, it is notable to mention the following germs: Proteus, Pseudomonas aeruginosa, Klebsiella, Ureaplasma urealyticum, which increase the $\mathrm{pH}$ of urine and produce 
urea. These bacteria can form infected stones or may form a mineral film-coating on a pre-existent stone, hiding a large number of germs, thus creating a bacterial tank which can be opened after an endoscopic intervention with an infectious episode and fever as complications [6]. All patients need to be considered infected until we have the laboratory results.

Because of the reduced number of patients (11 cases 6.7\%) with metabolic diseases (diabetes), associated with ureteral lithiasis which predisposed them to infections, we were unable to evaluate the association/risk of infections in the case of these patients.

Studies show that carrying out the urine culture exam does not represent a certain element of prediction for febrile cases in patients with endoscopic procedures. After obtaining the data regarding the dimension of the stones, correlated to the state of the secondary hydronephrosis, we can argue that in the cases of stones with smaller dimensions than $10 \mathrm{~mm}$, in which the febrile incidence was higher, the antibiotic prophylaxis determines the onset of fever in a lower number of patients compared to the ones that did not receive the antibiotics before the surgical procedures. Post operative urinary derivation plays a role in avoiding fever; therefore the catheterization of the urinary tract with double J ureteral catheter is advisable to prevent residual postoperative hydronephrosis and the onset of fever, particularly in the infected patients [7]. The analysis of the results in patients with grade II hydronephrosis, where the majority of the febrile cases occurred, shows an advantage in the patients who had a preoperative antibiotic treatment. The effects and effectiveness of antibiotic prophylaxis needs to be further analyzed in the case of patients with grade III hydronephrosis and stones larger than $10 \mathrm{~mm}$ on a larger group of patients. The results of the hemocultures performed at the onset of the febrile episode associated with shivers can provide further information in relation to the incriminated germ and its sensibility to antibiotics. The role of urine culture prior to an endoscopic intervention for ureteral lithiasis is important, even though it cannot objectify the exact location of the infection $[2,8]$. The need of sterilizing a urinary infection is crucial at this stage.

\section{Conclusions}

The evaluation of the urinary tract for possible infections (urine culture) is necessary preoperative, as well as the administration of a targeted antibiotic treatment in some cases. The preoperative administration of antibiotics can lead to a decrease in the frequency of infectious complications (fever, sepsis), reducing as a result the hospitalization days and other possible comorbidities.

\section{References}

1. Geavlete P. Ureteroscopia. Ed. Univ. „Carol Davila”, 2008, pg. 237-238.

2. EAU Indication for active stone removal and selection of procedure, Guidelines 2013 Edition, pg. 41-43.

3. EAU Perioperative antibacterial prophylaxis in Urology. Guidelines 2014 Edition, pg. 77-92.

4. Geavlete P, Jecu M, Geavlete B, et al: Ureteroscopy - an essential modern approach in upper urinary tract diagnosis and treatment. $J$ Med Life. 2010;3(2):193-199.

5. Geavlete P, Georgescu D, Niță G, Mirciulescu V, Cauni V. Complications of 2735 Retrograde Semirigid Ureteroscopy Procedures: A Single-Center Experience. Journal of Endourology. 2006;20(3):179-185. doi:10.1089/ end.2006.20.179.

6. Hakim NS, Paplois VE. Surgical complications: Diagnosis and treatment, Imperial College Press. 2007, pg. 587.

7. Merlo F, Cicerello E, Mangano M, Cova G, Maccatrozzo L. Stenting after ureteroscopy for ureteral lithiasis: results of a retrospective study. Arch Ital Urol Androl. 2011;83(1):57-9.

8. Mariappan P, Loong CW. Midstream urine culture and sensitivity test is a poor predictor of infected urine proximal to the obstructing ureteral stone or infected stones: a prospective clinical study. J Urol. 2004;171(6 Pt 1):2142-5. 